\title{
Erratum to: On weighted total least-squares with linear and quadratic constraints
}

\author{
Vahid Mahboub - Mohammad Ali Sharifi
}

Published online: 21 April 2013

(C) Springer-Verlag Berlin Heidelberg 2013

\section{Erratum to: J Geod (2013) 87: 279-286 \\ DOI 10.1007/s00190-012-0598-8}

In the afore-mentioned publication, Eqs. (19) and (30) contain some typos, which need to be corrected for. Eq. (19) should read

$$
\begin{aligned}
\frac{\partial \Phi}{\partial \xi} \mid & \tilde{\mathrm{e}}_{\mathrm{y}}, \tilde{\mathrm{e}}_{\mathrm{A}}, \hat{\lambda}_{1}, \hat{\lambda}_{2}, \hat{\lambda}_{3}, \hat{\xi} \\
& =-2\left(-A^{T} \hat{\lambda}_{1}+\tilde{\mathrm{E}}_{\mathrm{A}}^{\mathrm{T}} \hat{\lambda}_{1}+K^{T} \hat{\lambda}_{2}+M \hat{\xi} \hat{\lambda}_{3}\right)=0,
\end{aligned}
$$

and Eq. (30) should read

$$
\begin{aligned}
\hat{\lambda}_{2}= & {\left[K \mathrm{R}_{3} \mathrm{M} \hat{\xi} \hat{\xi}^{T} M^{T} \mathrm{R}_{3} \mathrm{~K}^{\mathrm{T}} / \hat{\xi}^{T} M \mathrm{R}_{3} M \hat{\xi}+\mathrm{KR}_{3} \mathrm{~K}^{\mathrm{T}}\right]^{-1} } \\
& \times\left[-K \mathrm{R}_{3} \mathrm{M} \hat{\xi} \hat{\xi}^{T} M^{T} \mathrm{R}_{3}\left(A^{\mathrm{T}} R_{1}+R_{2}\right) \mathrm{y} / \hat{\xi}^{T} M \mathrm{R}_{3} M \hat{\xi}\right. \\
& +a_{0}^{2} K \mathrm{R}_{3} \mathrm{M} \hat{\xi} / \hat{\xi}^{T} M \mathrm{R}_{3} M \hat{\xi} \\
& \left.+\mathrm{K}_{0}-\mathrm{KR}_{3}\left(A^{\mathrm{T}} R_{1}+R_{2}\right) \mathrm{y}\right]
\end{aligned}
$$

When taking these corrections into account, the proposed WTLS algorithm is as follows:

1st step: $[N, c]=A^{T} P_{y}[A, y], \xi^{(0)}=N^{-1} c$.

2nd step: For $i \in N$ compute:

The online version of the original article can be found under doi:10.1007/s00190-012-0598-8.

V. Mahboub ( $\square)$

Young Researchers Club, Lahijan Branch,

Islamic Azad University, Lahijan, Iran

e-mail: vahid_mahboobk@yahoo.com

M. A. Sharifi

Geodesy Division, Department of Geomatics Engineering,

Faculty of Engineering, Tehran University, Tehran, Iran

$$
\begin{aligned}
& R_{1}^{(\mathrm{i})}=\left[Q_{y}+\left(\hat{\xi}^{(i-1)^{\mathrm{T}}} \otimes I_{n}\right) Q_{A}\left(\hat{\xi}^{(i-1)} \otimes I_{n}\right)\right]^{-1} \\
& \hat{\lambda}_{1}^{(i)}=R_{1}^{(\mathrm{i})}\left(y-A \hat{\xi}^{(i-1)}\right) \\
& R_{2}^{(\mathrm{i})}=\left(I_{\mathrm{m}} \otimes \hat{\lambda}^{(i)^{T}}\right) Q_{A}\left(\hat{\xi}^{(i-1)} \otimes I_{n}\right) R_{1}^{(\mathrm{i})} \\
& R_{3}^{(\mathrm{i})}=\left(A^{\mathrm{T}} R_{1}^{(\mathrm{i})} A+R_{2}^{(\mathrm{i})} A\right)^{-1} \\
& \hat{\lambda}_{2}^{(i)}=\left[K R_{3}^{(\mathrm{i})} \mathrm{M} \hat{\xi}^{(i-1)} \hat{\xi}^{(i-1)^{T}} M^{T} R_{3}^{(\mathrm{i})} \mathrm{K}^{\mathrm{T}} / \hat{\xi}^{(i-1)^{T}} M R_{3}^{(\mathrm{i})} M \hat{\xi}^{(i-1)}\right. \\
& \left.+\mathrm{K} R_{3}^{(\mathrm{i})} \mathrm{K}^{\mathrm{T}}\right]^{-1} \times\left[-K R_{3}^{(\mathrm{i})} \mathrm{M} \hat{\xi}^{(i-1)} \hat{\xi}^{(i-1)^{T}} M^{T} R_{3}^{(\mathrm{i})}\left(A^{\mathrm{T}} R_{1}^{(\mathrm{i})}\right.\right. \\
& \left.+R_{2}^{(\mathrm{i})}\right) \mathrm{y} / \hat{\xi}^{(i-1)^{T}} M R_{3}^{(\mathrm{i})} M \hat{\xi}^{(i-1)} \\
& +a_{0}^{2} K R_{3}^{(\mathrm{i})} M \hat{\xi}^{(i-1)} / \hat{\xi}^{(i-1)^{T}} M R_{3}^{(\mathrm{i})} M \hat{\xi}^{(i-1)} \\
& \left.+\mathrm{K}_{0}-\mathrm{K} R_{3}^{(\mathrm{i})}\left(A^{\mathrm{T}} R_{1}^{(\mathrm{i})}+R_{2}^{(\mathrm{i})}\right) \mathrm{y}\right] \\
& \hat{\lambda}_{3}^{(i)}=\left[\hat { \xi } ^ { ( i - 1 ) } M R _ { 3 } ^ { ( \mathrm { i } ) } \left(\left(A^{\mathrm{T}} R_{1}^{(\mathrm{i})}+R_{2}^{(\mathrm{i})}\right) \mathrm{y}\right.\right. \\
& \left.\left.+K^{T} \hat{\lambda}_{2}^{(i)}\right)-a_{0}^{2}\right] / \hat{\xi}^{(i-1)^{T}} M R_{3}^{(\mathrm{i})} M \hat{\xi}^{(i-1)}
\end{aligned}
$$

$$
\hat{\xi}^{(i)}=R_{3}^{(\mathrm{i})}\left(\left(A^{\mathrm{T}} R_{1}^{(\mathrm{i})}+R_{2}^{(\mathrm{i})}\right) \mathrm{y}+K^{T} \hat{\lambda}_{2}^{(i)}+M \hat{\xi}^{(i-1)} \hat{\lambda}_{3}^{(i)}\right)
$$

3rd step: Repeat 2nd step until

$$
\left\|\hat{\xi}^{(i)}-\hat{\xi}^{(i-1)}\right\|<\delta
$$

for some chosen threshold $\delta$.

Unfortunately, a general proof of convergence of this algorithm cannot be given. Therefore, the statement made before Eq. (26) need to be modified. Instead of saying 
“... and due to the invertible property of $\left(A^{\mathrm{T}} R_{1} A+R_{2} A\right)$ (since $\left(A^{\mathrm{T}} R_{1} A\right)^{-1}$ exists) ...",

it should read:

“... and when assuming that the inverse of $\left(A^{\mathrm{T}} R_{1} A+R_{2} A\right)$ exists ...".
In example 3: rapid satellite positioning using the constrained WTLS algorithm as a regularized approach, we found that the unconstrained solution suffers from ill-conditioning; adding constraints has a regularizing effect. 